\title{
MPI in the era of CZT cameras: Absolute numbers are still better than relative figures
}

\author{
Riccardo Liga, $M D{ }^{a}$ and Alessia Gimelli, $M D^{b}$ \\ a Azienda Ospedaliero-Universitaria Pisana, Pisa, Italy \\ b Fondazione Toscana G. Monasterio, Pisa, Italy
}

Received May 27, 2019; accepted May 27, 2019

doi: $10.1007 / \mathrm{s} 12350-019-01777-\mathrm{x}$

\section{See related article, pp. 1072-1084}

In the last decades, the clinical characteristics of the patients that have been investigated by myocardial perfusion imaging (MPI) have changed dramatically, with a progressive reduction of the average cardiovascular risk and of the pre-test probability of coronary artery disease (CAD). ${ }^{1}$ These data have been recently confirmed by the results of some accurate retrospective analyses coming from leading institutions in the field of cardiac imaging, showing that in the current clinical practice the majority of patients undergoing nuclear cardiac imaging with single-photon emission computed tomography (SPECT) mainly report atypical symptoms and show a strikingly lower evidence of positive test results than in the past. ${ }^{2}$ Interestingly, those data were independent of the patients' clinical profile, with a disturbingly high prevalence of negative test results both in subjects with suspected and known CAD. ${ }^{2,3}$ Considering the strict interconnection between the accuracy of any ischemia test and the underlying prevalence of the disease, it is not surprising that the diagnostic capability of "standard" cardiac imaging methodologies has been decreasing substantially as compared to classical data. Those findings have been further confirmed by a comparative prospective cardiac imaging study for the diagnosis of patients with suspected CAD that demonstrated clearly that the accuracy of the major noninvasive functional imaging tests is, at best, in the range of $70 \%$, a rather modest result that can barely influence

Reprint requests: Alessia Gimelli, MD, Fondazione Toscana G. Monasterio, Pisa, Italy; gimelli@ftgm.it

J Nucl Cardiol 2021;28:1085-8.

1071-3581/\$34.00

Copyright (C) 2019 American Society of Nuclear Cardiology. patients' management. ${ }^{4}$ Among the major non-invasive myocardial imaging strategies, conventional SPECT seems to suffer relatively more from the changing of the patients' population, with relatively lower values of diagnostic accuracy than some other "competitors.", ,6 As an example, as reported in the recent "Cardiovascular magnetic resonance and single-photon emission computed tomography for diagnosis of coronary heart disease (CE-MARC)', trial, the sensitivity of MPI performed with traditional SPECT cameras in detecting obstructive CAD is significantly lower than that of cardiac magnetic resonance, ${ }^{5}$ although the clinical impact of such a difference is still disputed. ${ }^{7}$ Accordingly, an evolution of SPECT technology was urgently needed to maintain its long-standing status as an accurate and trusted strategy for the evaluation of CAD patients. The introduction of dedicated cardiac SPECT cameras equipped with cadmium-zinc-telluride (CZT) detectors has revolutionized nuclear cardiac imaging, as a technology that could allow a more accurate and faster evaluation of regional myocardial perfusion than standard Anger cameras. ${ }^{8}$ As a matter of fact, an increasing number of reports has demonstrated repeatedly that the increased photon sensitivity and spatial resolution of CZT pixelated crystals might overcome most of the technical limitation sodium-iodine (Na-I)-based devices, with superior diagnostic performances even in the most complex patients' categories, such as those with multivessel CAD. ${ }^{9,10}$ Moreover, CZT technology might allow investigating what is commonly regarded as the "holy Grail" of coronary pathophysiology, namely absolute myocardial blood flow (MBF) and myocardial perfusion reserve (MPR). ${ }^{11,12}$ In fact, absolute assessment of MBF is difficult with standard SPECT cameras, mainly because of the inadequate properties of the currently available flow tracers that show a non-linear extraction fraction at higher flow rates, coupled with the unfavorable physical properties of the Na-I crystals that typically saturate at higher count rates. CZT cameras 
allow image acquisition in a non-rotating mode and therefore acquisition of time-activity curves, which entail the computation of absolute myocardial perfusion parameters. ${ }^{12,13}$ As shown by a wide number of reports, the computation of myocardial perfusion parameters allows obtaining some of the most accurate predictors of $\mathrm{CAD}$, possibly individuating even subtle alterations of myocardial perfusion heterogeneity-as a possible result of sub-clinical and/or diffuse CAD, endothelial/microvascular dysfunction - that would be unapparent with a qualitative evaluation. ${ }^{13,14}$

Nevertheless, initial studies that have reported on the feasibility of absolute MBF with CZT technology have shown a systematic underestimation of flow values if compared with the available non-invasive and invasive gold standards, showing some of the possible limitations of this approach. ${ }^{12}$ Moreover, a direct comparison of the diagnostic capabilities of qualitative vs quantitative myocardial perfusion analysis at CZT is still lacking.

In their study, Nudi et al tried to compare the different capabilities of qualitative and quantitative myocardial perfusion assessment in patients with suspected or known CAD submitted to CZT imaging, with a particular reference on patient characteristics, imaging diagnostic yield, and short-term patients' outcome. ${ }^{15}$

To this purpose, a retrospective single-center evaluation of MPI data obtained with a dedicated cardiac CZT camera in 66 patients was performed. Patients underwent a single-day stress-rest CZT scan with sequential dynamic and static acquisitions and myocardial perfusion heterogeneity was evaluated both semiquantitatively and in absolute terms. Interestingly, a non-conventional semi-quantitative evaluation of regional myocardial perfusion was performed, using an alternative 7-region LV segmentation model previously reported by the same group of authors instead of the standardized 17-segment LV model. On the contrary, absolute MBF quantification was performed more traditionally and the average values of MBF and MPR for the 3 main coronary vascular territories were computed. According to the methodology, patients were followed up for major cardiovascular events for $\geq 1$ year, although no information on the event rate is provided.

The study mainly demonstrated the existence of a significant diagnostic discordance between the semiquantitative estimates of myocardial perfusion heterogeneity and absolute MBF and MPR values. In particular, only in 38 of the 66 enrolled patients (58\%) absolute and semi-quantitative perfusion analysis actually agreed, while in the remainders a discordant output was observed. This difference was mainly explained by the presence of a consistent number of patients with abnormal MPR values despite the absence of regional myocardial perfusion abnormalities on visual assessment. Interestingly, only limited impact of major demographical and clinical variables on myocardial perfusion variables was observed, with almost all the major cardiovascular risk factors having limited of insignificant association with any perfusion data.

Surprisingly, an abnormal LV perfusion on visual analysis appeared to be more strongly associated with the presence of obstructive CAD than absolute MBF, although no accuracy analysis was actually performed.

The authors should have commended for the CZT imaging protocol that tries to exploit all the advantages that this new technology offers in terms of superior architecture, photon sensitivity, and image resolution, which ultimately allow effective dynamic myocardial perfusion imaging.

Nevertheless, while the overall results of the study might be still of interest, a number of key limitation should also be considered for a comprehensive interpretation of the findings. First of all, it is not clear how the non-conventional LV segmentation model that was used actually takes into account the presence of myocardial scar, a parameter that, considering the clinical characteristics of the overall population ( $20 \%$ with previous myocardial infarction), could have influenced the results.

Moreover, it is strange that the 12 supposedly normal patients showed significant burden of inducible myocardial ischemia and scar, as demonstrated by elevated summed rest and difference scores $(2.6 \pm 1.5$ and $10.1 \pm 3.9$, respectively). Moreover, the curious finding that out of the total of 66 patients, no one presented anterior LV ischemia, and that only few (12\%) showed more than mild myocardial perfusion abnormalities poses doubts on the generalizability of the results.

In addition, saying that visual analysis was more accurate than absolute MBF quantification in detecting significant $\mathrm{CAD}$ is a countersense, since the presence of a significant reduction of stress MBF is what actually define a hemodynamically significant stenosis. It is likely that only patients with significant regional myocardial perfusion abnormalities were submitted to ICA, resulting in a referral bias that could have affected the diagnostic accuracy of myocardial perfusion variables in detecting angiographically significant CAD.

Nevertheless, the study by Nudi et al alluded to one of the most clinically relevant questions in modern nuclear cardiology: is there a difference in terms of CAD diagnosis and patient prognostication between absolute and relative measures of myocardial perfusion heterogeneity?

Regarding the diagnosis of significant CAD, solid evidence has shown the inconsistent relationship between stenosis anatomic severity and downstream MBF abnormalities, demonstrating that a combined 
assessment of CAD anatomic burden and regional myocardial perfusion is needed to evaluate patients with ischemic heart disease (IHD). ${ }^{14,16}$ In this context, absolute MBF quantification with positron emission tomography (PET) has been shown as the most accurate technique for the evaluation of the hemodynamic relevance of $\mathrm{CAD}$, and when combined with computed tomography coronary angiography (CTCA) through hybrid imaging it may entail a completely non-invasive coronary anatomic-functional analysis. ${ }^{17}$ In addition, the prognostic value of absolute MBF analysis in patients with IHD has been also accumulating, and recent reports have demonstrated how a relatively preserved coronary flow capacity associates with a favorable prognosis even in the presence of extensive CAD, while the presence of a more compromised absolute myocardial perfusion confers a dismal outcome, individuating patients that would benefit from aggressive therapeutic approaches (i.e., surgical coronary revascularization). ${ }^{18}$

Finally, the absolute quantification of myocardial perfusion has a capital role in the evaluation of patients with non-obstructed coronary arteries, in whom only MBF assessment may unmask the presence of functional alterations of coronary circulation (i.e., endothelial or microvascular dysfunction due to cardiovascular risk factors).$^{19}$ However, the limited availability of cardiac PET scanners and the intrinsic technical complexity of the methodology have prevented the diffusion of absolute MBF evaluation in daily clinical practice.

The introduction of dedicated cardiac CZT camera has represented a technological breakthrough in the field, giving also the chance to perform dynamic cardiac acquisitions in the context of a clinically indicated myocardial perfusion scan. ${ }^{11-13}$ Accordingly, initial evidence has confirmed the feasibility of MBF quantification on CZT cameras, allowing mirroring the results obtained by PET imaging and predicting the presence of flow-limiting CAD as evaluated by invasive fractional flow reserve (FFR). ${ }^{12}$ In particular, while the evaluation of relative myocardial perfusion may still remain the cornerstone for the diagnosis of CAD in the majority of patients, absolute MBF analysis may prove particularly beneficial in patients with extensive CAD burden, in whom semi-quantitative measures may fail to unmask coronary involvement and possibly predict appropriately the risk of future cardiac events. ${ }^{20}$ At the opposite extreme, patients with subtle myocardial perfusion abnormalities on semi-quantitative CZT analysis but preserved regional MBF and MPR could be reasonably spared from any further diagnostic test and invasive therapy, because of the superior prognostic impact of optimal medical therapy in this setting.

CZT devices may thus represent the long-awaited innovation in the field of MPI, regenerating the interest for a methodology that had recently suffered the competition with some other competing imaging modalities. The evaluation of absolute MBF and MPR can be seen as the "ace in the hole" of CZT MPI, allowing investigating the most intimate aspects of coronary pathophysiology in an entirely non-invasive manner at the cost of an extremely contained radiation burden. ${ }^{11-13}$ In this context, while global MBF and MPR are among the most powerful prognosticators in cardiac patients, ${ }^{14}$ the assessment of patients with IHD requires the computation of regional myocardial perfusion data, in order to individuate more subtle alterations of MBF distribution that might indicate the presence of intermediate coronary lesions that might benefit from coronary revascularization. ${ }^{20}$

\section{Disclosure}

Drs. Liga and Gimelli have nothing to disclose.

\section{References}

1. Cheng VY, Berman DS, Rozanski A, Dunning AM, Achenbach S, Al-Mallah M, et al. Performance of the traditional age, sex, and angina typicality-based approach for estimating pretest probability of angiographically significant coronary artery disease in patients undergoing coronary computed tomographic angiography: results from the multinational coronary $\mathrm{CT}$ angiography evaluation for clinical outcomes: an international multicenter registry (CONFIRM). Circulation 2011;124:2423-32.

2. Rozanski A, Gransar H, Hayes SW, Min J, Friedman JD, Thomson LE, et al. Temporal trends in the frequency of inducible myocardial ischemia during cardiac stress testing: 1991 to 2009. J Am Coll Cardiol 2013;61:1054-65.

3. Jouni H, Askew JW, Crusan DJ, Miller TD, Gibbons RJ. Temporal trends of single-photon emission computed tomography myocardial perfusion imaging in patients with coronary artery disease: A 22-year experience from a tertiary academic medical center. Circ Cardiovasc Imaging 2017;10:e005628.

4. Neglia D, Rovai D, Caselli C, Pietila M, Teresinska A, AguadéBruix S, et al. Detection of significant coronary artery disease by noninvasive anatomical and functional imaging. Circ Cardiovasc Imaging 2015;8:e02179.

5. Greenwood JP, Maredia N, Younger JF, Brown JM, Nixon J, Everett $\mathrm{CC}$, et al. Cardiovascular magnetic resonance and single-photon emission computed tomography for diagnosis of coronary heart disease (CE-MARC): A prospective trial. Lancet 2012;379:453-60.

6. Danad I, Raijmakers PG, Driessen RS, Leipsic J, Raju R, Naoum C, et al. Comparison of coronary CT angiography, SPECT, PET, and hybrid imaging for diagnosis of ischemic heart disease determined by fractional flow reserve. JAMA Cardiol 2017;2:1100-7.

7. Greenwood JP, Ripley DP, Berry C, McCann GP, Plein S, Bucciarelli-Ducci $\mathrm{C}$, et al. Effect of care guided by cardiovascular magnetic resonance, myocardial perfusion scintigraphy, or NICE guidelines on subsequent unnecessary angiography rates: The CEMARC 2 randomized clinical trial. JAMA 2016;316:1051-60.

8. Gimelli A, Bottai M, Giorgetti A, Genovesi D, Kusch A, Ripoli A, et al. Comparison between ultrafast and standard single-photon emission CT in patients with coronary artery disease: A pilot study. Circ Cardiovasc Imaging 2011;4:51-8. 
9. Gimelli A, Liga R, Duce V, Kusch A, Clemente A, Marzullo P. Accuracy of myocardial perfusion imaging in detecting multivessel coronary artery disease: A cardiac CZT study. J Nucl Cardiol 2017;24:687-95.

10. Gimelli A, Bottai M, Giorgetti A, Genovesi D, Filidei E, Marzullo P. Evaluation of ischaemia in obese patients: feasibility and accuracy of a low-dose protocol with a cadmium-zinc telluride camera. Eur J Nucl Med Mol Imaging 2012;39:1254-61.

11. Agostini D, Roule V, Nganoa C, Roth N, Baavour R, Parienti JJ, et al. First validation of myocardial flow reserve assessed by dynamic 99mTc-sestamibi CZT-SPECT camera: head to head comparison with 15O-water PET and fractional flow reserve in patients with suspected coronary artery disease. The WATERDAY study. Eur J Nucl Med Mol Imaging 2018;45:1079-90.

12. Zavadovsky KV, Mochula AV, Boshchenko AA, Vrublevsky AV, Baev AE, Krylov AL, et al. Absolute myocardial blood flows derived by dynamic CZT scan vs invasive fractional flow reserve: Correlation and accuracy. J Nucl Cardiol 2019. https://doi.org/10. 1007/s12350-019-01678-z.

13. Ben Bouallègue F, Roubille F, Lattuca B, Cung TT, Macia JC, Gervasoni R, et al. SPECT myocardial perfusion reserve in patients with multivessel coronary disease: Correlation with angiographic findings and invasive fractional flow reserve measurements. J Nucl Med 2015;56:1712-7.

14. Gould KL, Johnson NP, Bateman TM, Beanlands RS, Bengel FM, Bober R, et al. Anatomic versus physiologic assessment of coronary artery disease. Role of coronary flow reserve, fractional flow reserve, and positron emission tomography imaging in revascularization decision-making. J Am Coll Cardiol 2013;62:1639-53.

15. Nudi F, Biondi-Zoccai G, Nudi A, Neri G, Procaccini E, Schilllaci O. Comparative analysis between myocardial perfusion reserve and maximal ischemia score at single photon emission computed tomography with newgeneration cadmium-zinc-telluride cameras. J Nucl Cardiol (in press)

16. Liga R, Vontobel J, Rovai D, Marinelli M, Caselli C, Pietila M, et al. Multicentre multi-device hybrid imaging study of coronary artery disease: results from the EValuation of INtegrated Cardiac Imaging for the Detection and Characterization of Ischaemic Heart Disease (EVINCI) hybrid imaging population. Eur Heart J Cardiovasc Imaging 2016;17:951-60.

17. Kajander S, Joutsiniemi E, Saraste M, Pietilä M, Ukkonen H, Saraste A, et al. Cardiac positron emission tomography/computed tomography imaging accurately detects anatomically and functionally significant coronary artery disease. Circulation 2010;122:603-13.

18. Gould KL, Johnson NP, Roby AE, Nguyen T, Kirkeeide R, Haynie $\mathrm{M}$, et al. Regional, artery-specific thresholds of quantitative myocardial perfusion by PET associated with reduced myocardial infarction and death after revascularization in stable coronary artery disease. J Nucl Med 2019;60:410-7.

19. Neglia D, Sampietro T, Vecoli C, Liga R, Rossi G, Filidei E, et al. Abnormal glucose and lipid control in non-ischemic left ventricular dysfunction. J Nucl Cardiol 2012;19:1182-9.

20. Hajjiri MM, Leavitt MB, Zheng H, Spooner AE, Fischman AJ, Gewirtz H. Comparison of positron emission tomography measurement of adenosine-stimulated absolute myocardial blood flow versus relative myocardial tracer content for physiological assessment of coronary artery stenosis severity and location. JACC Cardiovasc Imaging 2009;2:751-8.

Publisher's Note Springer Nature remains neutral with regard to jurisdictional claims in published maps and institutional affiliations. 